and remained at a location susceptible to aspiration. The patient went on to suffer acute respiratory failure as a result of acute pneumonitis and eventually had a tracheostomy tube inserted. This demonstrates the importance of aspiration precautions for patients with ileus.

@ERSpublications

Risk of aspiration needs to be accounted for in patients with ileus who have had oral contrast ingestion http://ow.ly/vyKw3

Naseem Helo, Daniel Hoss and Mark Girguis

Dept of Radiology, Loma Linda University Medical Center, Loma Linda, CA, USA.

Correspondence: Naseem Helo, Dept of Radiology, Loma Linda University Medical Center, 11234 Anderson St, Loma Linda, 92354 CA, USA. E-mail: Nhelo@llu.edu

Received: March 132014 | Accepted: March 172014

Conflict of interest: None declared.

Provenance: Submitted article, peer-reviewed.

\title{
An unexpected discovery complicating sicca syndrome
}

During a routine check-up, retro-pneumoperitoneum was discovered on the lowest slices of a computed tomography (CT) chest scan in a 77-year-old female patient who was known to have Sjögren's syndrome with lymphocytic interstitial pneumonia (fig. 1a). She had initially been treated with prednisolone that improved sicca signs and cough. The patient, who had no prior history of digestive problems, complained of abdominal discomfort and distension. She was afebrile, haemodynamically stable and bowel movement was unchanged. Abdominal examination eliminated signs of peritonism.

The abdominal CT scan, which showed thickening of the wall of the descending colon, contained multiple air-filled cysts (black arrows) consistent with pneumatosis cystoides intestinalis (PCI) (fig. 1b). There was free air in the wall of the caecum, colon and adjacent mesos (fig. 1c).

PCI is a rare condition characterised by multilocular pneumatocysts within the wall of the lower digestive tract. To date, association with a systemic connective tissue disease (CTD) such as Sjögren's syndrome has only been reported twice [1]. Previous studies have mostly been of scleroderma-related conditions [2]. Corticosteroids are often used in CTD and appear to increase the risk of PCI [3]. Conservative treatment is often sufficient in stable patients with limited symptoms. Antibiotic agents (e.g. metronidazole) and the tapering of immunosuppressive drugs seem to improve clinical and radiological findings. Intestinal rest, parenteral nutrition, electrolyte supplementation and inhaled oxygen are also recommended [3].
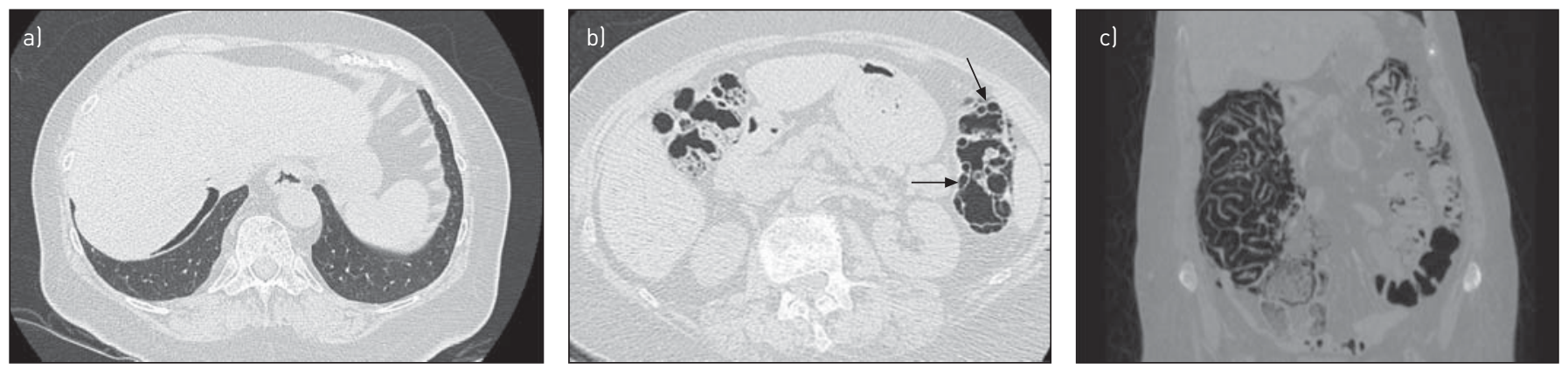

FIGURE 1 a) Computed tomography (CT) chest scan showing retro-pneumoperitoneum. b) Abdominal CT scan showing thickening of the wall of the descending colon, which contained multiple air-filled cysts (black arrows). c) Coronal CT reconstruction showing free air in the wall of the caecum, colon and adjacent mesos. 
Unnecessary surgery can be avoided through better knowledge of PCI and its complications. Surgical intervention should only be considered in severe or life-threatening cases.

(2) @ERSpublications

Awareness of pneumatosis cystoides intestinalis with Sjögren's syndrome could avoid unnecessary treatment escalation http://ow.ly/ubeaD

Nihal Martis ${ }^{1}$, Marie Baque-Juston ${ }^{2}$, Matthieu Buscot ${ }^{1}$, Charles-Hugo Marquette ${ }^{1}$ and Sylvie Leroy $^{1}$

${ }^{1}$ Dept of Respiratory Medicine, University Hospital, Nice, France. ${ }^{2}$ Dept of Radiology, University Hospital, Nice, France.

Correspondence: Nihal Martis, University Hospital of Nice, C.H.U. Pasteur, 30 avenue de la Voie Romaine, CS 51069, 06002 Nice, France. E-mail: martis.n@chu-nice.fr

Received: Jan 052014 | Accepted: Jan 082014

Conflict of interest: None declared.

Provenance: Submitted article, peer reviewed.

\section{References}

1 Vandergheynst F, Van Gansbeke D, Steinfeld S. Primary Sjögren's syndrome associated with pneumatosis cystoides coli. Eur J Intern Med 2006; 17: 57-58.

2 Balbir-Gurman A, Brook OR, Chermesh I, et al. Pneumatosis cystoides intestinalis in scleroderma-related conditions. Intern Med J 2012; 42: 323-329.

3 Zhang H, Jun SL, Brennan TV. Pneumatosis intestinalis: not always a surgical indication. Case Rep Surg 2012; 2012: 719713 .

Eur Respir Rev 2014; 23: 397-398 | DOI: 10.1183/09059180.00000114 | Copyright @ERS 2014 ERR articles are open access and distributed under the terms of the Creative Commons Attribution Non-Commercial Licence 4.0. 